\title{
Low Ten-eleven-translocation 2 (TET2) transcript level is independent of TET2 mutation in patients with myeloid neoplasms
}

Renata Scopim-Ribeiro 1,2, João Agostinho Machado-Neto ${ }^{1,2}$, Paula de Melo Campos ${ }^{1}$, Fernanda Soares Niemann', Irene Lorand-Metze ${ }^{1}$, Fernando Ferreira Costa ${ }^{1}$, Sara Teresinha Olalla Saad ${ }^{1}$ and Fabiola Traina ${ }^{1,2^{*}}$

\begin{abstract}
Background: New sequencing technologies have enabled the identification of mutations in Ten-eleven-translocation 2 (TET2), an enzyme that catalyzes the conversion of 5-methylcytosine into 5-hydroxymethylcytosine (5-hmC) in myeloid neoplasms. We have recently identified reduced TET2 mRNA expression in myelodysplastic syndromes (MDS) and acute myeloid leukemia (AML), which is associated with a poor overall survival in MDS. We herein aimed to investigate TET2 mutations and their impact on TET2 expression in a cohort of patients with myeloid neoplasms, including MDS and AML patients.
\end{abstract}

Findings: TET2 mutations were observed in 8 out of 19 patients (42\%) with myeloid neoplasms. The TET2 expression profile was similar between in wild type and in TET2 mutated patients.

Conclusion: Our results suggest that TET2 expression is reduced in MDS/AML patients, independently of mutational status.

Keywords: Ten-eleven-translocation 2, TET2, Mutation, Myelodysplastic syndromes, Acute myeloid leukemia

\section{Findings} Introduction

New sequencing technologies have enabled the identification of several mutations in epigenetic regulator genes, such as Ten-eleven-translocation 2 (TET2), which encodes an enzyme that catalyzes the conversion of 5methylcytosine into 5-hydroxymethylcytosine (5-hmC). Mutations in the coding regions of TET2 lead to loss of TET2 protein function, reduce 5 -hmc levels and cause a significant shift towards monocyte/granulocyte differentiation in detriment of erythroid/lymphoid differentiation [1,2]. TET2 mutations confer a worse prognosis in myelodysplastic syndromes (MDS) and acute myeloid leukemia (AML) $[3,4]$. We have recently reported that

\footnotetext{
* Correspondence: ftraina@fmrp.usp.br

${ }^{1}$ Hematology and Hemotherapy Center, University of Campinas/

Hemocentro-Unicamp, Instituto Nacional de Ciência e Tecnologia do

Sangue, Rua Carlos Chagas, 480, CEP 13083-878, Campinas, SP, Brazil

${ }^{2}$ Present address: Department of Internal Medicine, University of São Paulo at

Ribeirão Preto Medical School, Ribeirão Preto, SP, Brazil
}

TET2 mRNA expression is downregulated in MDS and AML, and predicts poor overall survival in MDS patients [5]. However, the correlation between the presence of TET2 mutations and TET2 transcript levels has rarely been addressed $[4,6]$. We, herein, expanded our previous observations and aimed to investigate TET2 mutational status and the impact of this mutational status on TET2 expression in a cohort of MDS and AML patients. For this purpose, TET2 mutation analysis and TET2 gene expression were tested by Sanger sequencing and quantitative PCR, respectively, in bone marrow samples from healthy donors and myeloid neoplasm patients.

\section{Materials and methods \\ Patients' characteristics}

Bone marrow samples were obtained from a total of 22 healthy donors, and 19 patients with myeloid neoplasms $(\mathrm{MDS}=10$ and $\mathrm{AML}=9)$ followed at the outpatient clinics of the University of Campinas, who had also been 
included in a previous study [5]. The present study was approved by the Institutional and National Review Board in accordance with the Helsinki Declaration. Written informed consent was obtained from all patients who participated in this study. Patients were diagnosed according to the 2008 Word Health Organization criteria [7]. Patients' characteristics are described in Table 1.

\section{Polymerase chain reaction $(P C R)$ and DNA sequencing}

The screening of TET2 mutations was performed on coding exons 3, 4, 5, 6, 7, 8, 9, 10 and 11 (GenBank reference NM_0175628.4). Primer sequences and PCR

Table 1 Patients' characteristics

\begin{tabular}{|c|c|}
\hline Patients & Number \\
\hline MDS & 10 \\
\hline \multicolumn{2}{|l|}{ Gender } \\
\hline Male/Female & $5 / 5$ \\
\hline Age (years), median (range): & $58(28-78)$ \\
\hline \multicolumn{2}{|l|}{ WHO 2008} \\
\hline RA & 2 \\
\hline RARS & 1 \\
\hline RCMD & 4 \\
\hline RAEB-1 & 3 \\
\hline \multicolumn{2}{|l|}{ IPSS-R } \\
\hline Low & 5 \\
\hline Intermediate & 2 \\
\hline High & 1 \\
\hline Not available & 2 \\
\hline \multicolumn{2}{|l|}{ Cytogenetics } \\
\hline Normal karyotype & 10 \\
\hline \multicolumn{2}{|l|}{ BM blast (\%) } \\
\hline$<5 \%$ & 7 \\
\hline$\geq 5$ and $<10 \%$ & 3 \\
\hline AML & 9 \\
\hline \multicolumn{2}{|l|}{ Gender } \\
\hline Male/Female & $4 / 5$ \\
\hline Age (years), median (range) & $66(44-90)$ \\
\hline BM blasts (\%), median (range) & $92(31-98.5)$ \\
\hline \multicolumn{2}{|l|}{ Cytogenetics } \\
\hline Normal karyotype & 2 \\
\hline Deletion $5 q$ & 1 \\
\hline Monosomy 7 & 1 \\
\hline Complex karyotype & 1 \\
\hline No growth & 4 \\
\hline
\end{tabular}

Abbreviations: MDS myelodysplastic syndromes, WHO World Health Organization, $R A$ refractory anemia, RARS refractory anemia with ringed sideroblasts, del( $5 q)$ MDS with isolated del( $5 q), R C M D$ refractory cytopenia with multilineage dysplasia, RAEB-1 refractory anemia with excess blast-1, BM bone marrow, IPSS-R Revised International Prognostic Scoring System, AML acute myeloid leukemia conditions were previously described [8]. Amplicons were sequenced with an ABI 3500 Genetic Analyzer (Life Technologies, Carlsbad, CA, USA) using the Big Dye terminator V1.1 cycle sequencing kit and analyzed using CLC Main Workblench v.7.6.2 software (Qiagen, Aarhus, Denmark). All alterations found were searched in SNP (dbSNP; http:// www.ncbi.nlm.nih.gov/projects/SNP), Ensembl Genome Browser databases (http://asia.ensembl.org/index.html), and in the Catalogue of Somatic Mutations in Cancer (COSMIC; http://cancer.sanger.ac.uk/cosmic).

\section{Quantitative PCR ( $q P C R$ )}

Total RNA was extracted from cells using the TRIzol reagent (Life Technologies, Carlsbad, CA, USA). The reverse transcription reaction was performed using RevertAid $^{\text {Tw }}$ First Strand cDNA Synthesis Kit (MBI Fermentas, St. Leon-Rot, Germany). TET2 mRNA level was detected by Maxima Sybr green qPCR master mix (MBI Fermentas, St. Leon-Rot, Germany) in the ABI 7500 Sequence Detection System (Life Technologies) using specific primers: forward 5' - ACGCAAGCCAGGCTAA ACA -3', reverse 5'- GCTGGGACTGCTGCATGA -3'; HPRT1 (hypoxanthine phosphoribosyltransferase 1) was used as the endogenous control: forward 5'-GAACGT CTTGCTCGAGATGTGA-3', reverse 5'-TCCAGCAGG TCAGCAAAGAAT-3'. The relative quantification value was calculated using the equation, $2^{-\Delta \Delta C T}$ [9]. A negative 'No Template Control' was included for each primer pair. The dissociation protocol was performed at the end of each run to check for non-specific amplifications. Three replicas were run on the same plate for each sample.

\section{Statistical analyses}

Statistical analyses were performed using GraphPad Instat 5 (GraphPad Software, Inc., San. Diego, CA, USA). For comparisons, Mann-Whitney test was used for measured factors with two levels. A $p$ value $<0.05$ was considered as statistically significant.

\section{Results and discussion}

We analyzed the mutation status of TET2 exons and the impact of this status on TET2 expression in bone marrow cells from myeloid neoplasm patients. In total, 17 TET2 variants were detected. After excluding confirmed SNPs (P29R [rs12498609], V218M [rs6843141], P363L [rs17253 672], G355D [rs61744960], H1778R [rs62621450], I1762V [rs2454206] and L1721W [rs34402524]), TET2 mutations were observed in 8/19 (42 \%) patients with myeloid neoplasms [4/10 (40 \%) MDS and 4/9 (44\%) AML], including six missense (E709K, Y867H, H924R, S1109P, P1723S and H1868L) and three stop codon (E1073X, S1516X and S1518X) mutations (Fig. 1). A total of nine TET2 mutations were found in exon $3(n=5)$ or exon 11 $(n=3)$ in eight patients; one patient had two mutations. 


Fig. 1 TET2 mutations identified in myelodysplastic syndromes and acute myeloid leukemia patients. In a cohort of 19 patients, nine TET2 mutations were
identified in eight patients. Genomic sequencing of protein-COding regions revealed missense (black arrows), and stop codon (red arrows) mutations in
TET2; Sanger sequencing analysis is illustrated in the figure. TET2 protein primary structure indicating the domains and specific known conserved motifs are
shown: cysteine-rich region (C-rich), double strand beta helix (DSBH). The aminoacid position is indicated
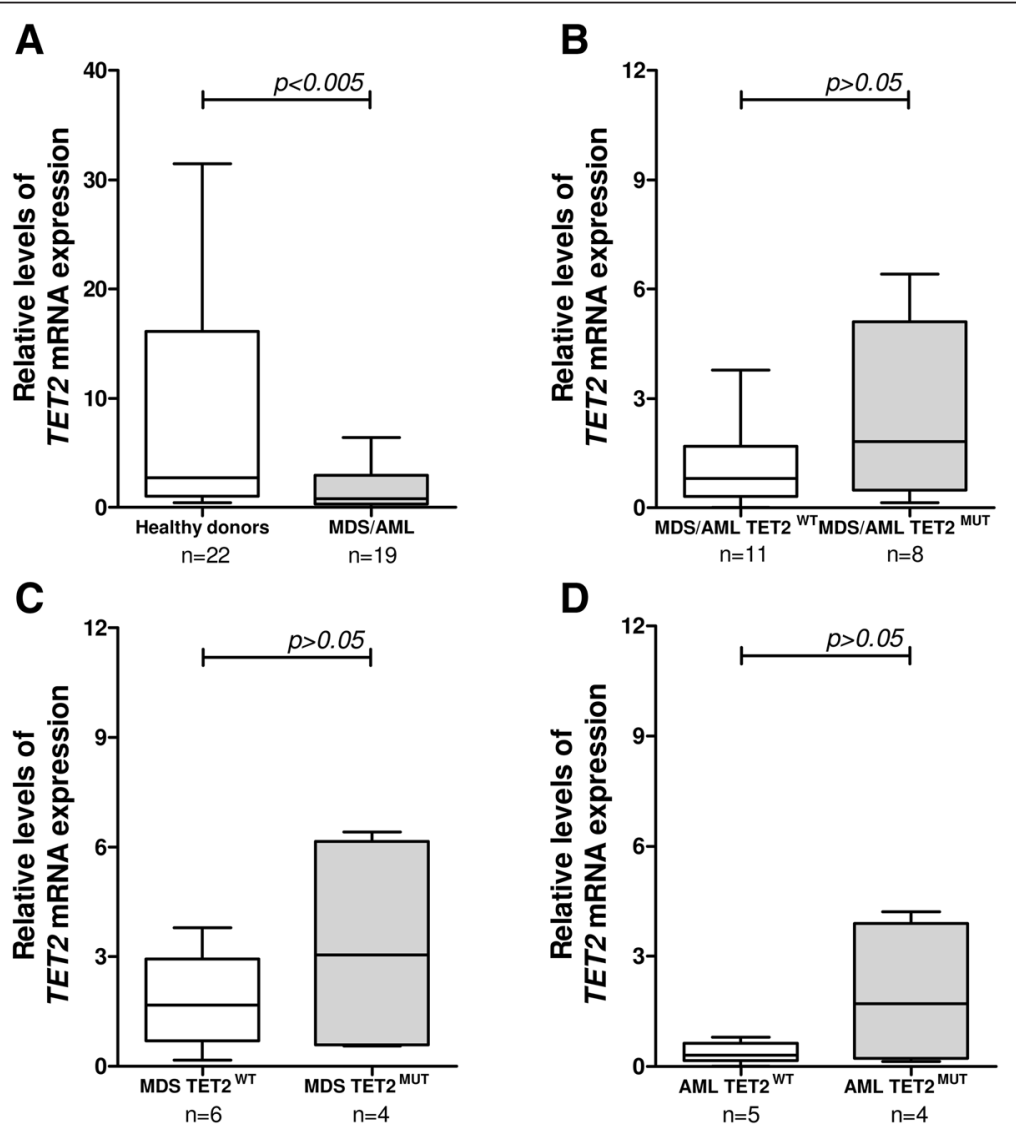

Fig. 2 TET2 expression, according to TET2 mutational status, in myelodysplastic syndromes and acute myeloid leukemia patients. a qPCR analysis of Ten-eleven-translocation 2 (TET2) mRNA levels in total bone marrow cells from healthy donors and from patients with the diagnosis of myelodysplastic syndromes (MDS)/acute myeloid leukemia (AML). TET2 expression in patients stratified according to TET2 mutational status for the entire cohort of MDS/ AML patients (b), for patients with MDS (c) or AML diagnosis (d). The "y" axis represents the relative TET2 mRNA expression. The numbers of subjects studied and the $p$ values (Mann-Whitney) are indicated in the graph. Abbreviations: WT wild type, MUT mutated 
Four TET2 mutations (E1073X, S1109P, S1518X and H1868L) found in our study had not been previously described in the COSMIC database. Among the six missense mutations, three mutations presented a high probably of damage to protein function, according to Polyphen2 analysis. The PolyPhen2 scores for predicted damage of TET2 Y867H, S1109P and H1868L mutations were 0.999, 0.995 and 1, respectively (http://genetics.bwh.harvard.edu/pph2/). TET2 mutations in exons have been implicated in protein loss-of-function and myeloid neoplasm mechanism of disease [1, 2], which supports our focus on sequencing TET2 coding regions only. The relevance of mutations in the TET2 promoter region has not been explored yet in myeloid neoplasms.

TET2 expression was reduced in MDS/AML patients (median 0.8 [minimum 0.01- maximum 6.41]), compared with healthy donors (2.72 [0.43-31.49]); $p<0.005$ (Fig. 2a). The standard deviation of TET2 expression for the healthy donors was high in our cohort of patients, which is in agreement with reports from other authors $[4,10]$. TET2 expression was similar in TET2 wild type (0.8 [0.01-3.79]) and in mutated patients (1.82 [0.14-6.41]), for the entire cohort, and among patients with MDS and AML only, all $p>0.05$ (Fig. $2 \mathrm{~b}-\mathrm{d}$ ). These findings are in agreement with Jankowska and colleagues [4] and Coutinho and colleagues [6], who reported TET2 downregulation in $16 \mathrm{pa-}$ tients with MDS/myeloproliferative neoplasms and in 12 patients with pediatric MDS, respectively, regardless of TET2 mutational status. Reduced TET2 levels in MDS patients have also been previously reported by our group [5], Li and collaborators [10], and Zhang and collaborators [11]. However, the TET2 mutation was not evaluated in these studies.

Somatic mutations in TET2 have been described in healthy elderly individuals with clonal hematopoiesis and have been associated with alterations in DNA methylation [12]. TET2 mutations have been indicated as early events in clonal evolution and potently lead to the development of preleukemic hematopoietic stem cells $[13,14]$. For this reason, growing attention has been given to the hypothesis that TET2 is a gate keeper in hematological malignancies. The occurrence of TET2 mutations may indirectly lead to the disruption of other important genes by inducing epigenetic changes, as recently described for JAK2 [15]. TET2 mutations alter the transcriptional consequences of JAK2 V617F in a cell-intrinsic manner and prevent JAK2 V617F from up-regulating a proliferative program [15]. In addition, Kameda et al. [16] and Rasmussen and collaborators [17] demonstrated, using mouse models, that the loss of Tet 2 contributes towards the progression of myeloid neoplasms. Thus, reduced TET2 function, due to loss-offunction mutations, reduced mRNA expression due to epigenetic silencing or not yet elucidated mechanisms may have a great contribution to the malignant phenotype of hematological cancers.

Taken together, our findings suggest that decreased TET2 expression is observed in myeloid neoplasms and does not correlate with TET2 mutation status. Other molecular mechanisms, including mutations of additional genes and/or epigenetic regulation may affect TET2 expression in myeloid neoplasms [18]. TET2 mRNA expression levels, in addition to TET2 mutation status, may also play a role in myeloid neoplasm physiopathology. Future studies to further investigate the mechanisms that lead to reduced TET2 expression in hematological malignancies will be of importance.

\begin{abstract}
Abbreviations
5-hmC: 5-hydroxymethylcytosine; AML: acute myeloid leukemia; COSMIC: catalogue of somatic mutations in cancer; HPRT1: hypoxanthine phosphoribosyltransferase 1; JAK2: Janus kinase 2; MDS: myelodysplastic syndromes; PCR: polymerase chain reaction; qPCR: quantitative PCR; SNPs: single nucleotide polymorphisms; TET2: ten-eleven-translocation 2.
\end{abstract}

\section{Competing interests}

The authors declare that they have no competing interests.

\section{Authors' contributions}

RSR designed the study and experiments, performed all the experiments, statistical analyses, patient database, manuscript preparation, completion and final approval. JAMN participated in the analysis of the data, quantitative PCR experiments, manuscript preparation and final approval. PMC participated in the interpretation of manuscript data, clinical data collection, manuscript editing, and final approval. FSN participated in sequencing experiments. ILM revised the diagnoses and together with FFC participated in the manuscript editing and final approval. STOS participated in patient follow up, manuscript editing and final approval. FT participated in the overall design of the study and experiments, statistical analyses, patient follow up, manuscript preparation, editing, completion and final approval. All authors read and approved the final manuscript.

\section{Acknowledgments}

The authors would like to thank Dr. Nicola Conran and Raquel S Foglio for English review, and Tereza Salles for her valuable technical assistance. This work was supported by Conselho Nacional de Desenvolvimento Científico e Tecnológico (CNPq), Fundação de Amparo à Pesquisa do Estado de São Paulo (FAPESP). Grant numbers: 610021/2009-5; 2011/51959-0; 2011/15905-3; 2012/ 09982-8.

Received: 16 November 2015 Accepted: 29 February 2016

Published online: 16 March 2016

\section{References}

1. Ko M, Huang Y, Jankowska AM, Pape UJ, Tahiliani M, Bandukwala HS, et al. Impaired hydroxylation of 5-methylcytosine in myeloid cancers with mutant TET2. Nature. 2010;468:839-43.

2. Pronier E, Almire C, Mokrani H, Vasanthakumar A, Simon A, da Costa Reis Monte Mor B, et al. Inhibition of TET2-mediated conversion of 5-methylcytosine to 5-hydroxymethylcytosine disturbs erythroid and granulomonocytic differentiation of human hematopoietic progenitors. Blood. 2011;118:2551-5.

3. Delhommeau F, Dupont S, Della Valle V, James C, Trannoy S, Masse A, et al. Mutation in TET2 in myeloid cancers. N Engl J Med. 2009;360:2289-301.

4. Jankowska AM, Szpurka H, Tiu RV, Makishima H, Afable M, Huh J, et al. Loss of heterozygosity $4 \mathrm{q} 24$ and TET2 mutations associated with myelodysplastic/myeloproliferative neoplasms. Blood. 2009;113:6403-10.

5. Scopim-Ribeiro R, Machado-Neto JA, Campos Pde M, Silva CA, Favaro P Lorand-Metze I, et al. Ten-Eleven-Translocation 2 (TET2) is downregulated in myelodysplastic syndromes. Eur J Haematol. 2015;94:413-8.

6. Coutinho DF, Monte-Mor BC, Vianna DT, Rouxinol ST, Batalha AB, Bueno AP, et al. TET2 expression level and 5-hydroxymethylcytosine are decreased in refractory cytopenia of childhood. Leuk Res. 2015;39:1103-8. 
7. Swerdlow SH, Campo E, Harris NL, Jaffe ES, Pileri SA, Stein H, et al. WHO classification of tumours of haematopoietic and lymphoid tissues. 4th ed. Lyon: IARC; 2008.

8. Traina F, Visconte V, Elson P, Tabarroki A, Jankowska AM, Hasrouni E, et al. Impact of molecular mutations on treatment response to DNMT inhibitors in myelodysplasia and related neoplasms. Leukemia. 2014;28:78-87.

9. Livak KJ, Schmittgen TD. Analysis of relative gene expression data using real-time quantitative PCR and the 2(-Delta Delta C(T)) Method. Methods. 2001;25:402-8

10. Li S, Fan R, Zhao XL, Wang XQ. CXXC4 mRNA levels are associated with clinicopathological parameters and survival of myelodysplastic syndrome patients. Leuk Res. 2014;38:1072-8.

11. Zhang W, Shao ZH, Fu R, Wang HQ, Li LJ, Wang J, et al. TET2 expression in bone marrow mononuclear cells of patients with myelodysplastic syndromes and its clinical significances. Cancer Biol Med. 2012:9:34-7.

12. Busque L, Patel JP, Figueroa ME, Vasanthakumar A, Provost S, Hamilou Z, et al. Recurrent somatic TET2 mutations in normal elderly individuals with clonal hematopoiesis. Nat Genet. 2012;44:1179-81.

13. Jan M, Snyder TM, Corces-Zimmerman MR, Vyas P, Weissman IL, Quake SR et al. Clonal evolution of preleukemic hematopoietic stem cells precedes human acute myeloid leukemia. Sci Transl Med. 2012;4:149ra118.

14. Itzykson R, Kosmider O, Renneville A, Morabito M, Preudhomme C, Berthon $C$, et al. Clonal architecture of chronic myelomonocytic leukemias. Blood. 2013;121:2186-98

15. Ortmann CA, Kent DG, Nangalia J, Silber Y, Wedge DC, Grinfeld J, et al. Effect of mutation order on myeloproliferative neoplasms. N Engl J Med. 2015;372:601-12

16. Kameda T, Shide K, Yamaji T, Kamiunten A, Sekine M, Taniguchi Y, et al. Loss of TET2 has dual roles in murine myeloproliferative neoplasms: disease sustainer and disease accelerator. Blood. 2015;125:304-15.

17. Rasmussen KD, Jia G, Johansen JV, Pedersen MT, Rapin N, Bagger FO, et al. Loss of TET2 in hematopoietic cells leads to DNA hypermethylation of active enhancers and induction of leukemogenesis. Genes Dev. 2015;29:910-22.

18. Wang $Y$, Xiao M, Chen $X$, Chen L, Xu Y, Lv L, et al. WT1 recruits TET2 to regulate its target gene expression and suppress leukemia cell proliferation. Mol Cell. 2015;57:662-73.

\section{Submit your next manuscript to BioMed Central and we will help you at every step:}

- We accept pre-submission inquiries

- Our selector tool helps you to find the most relevant journal

- We provide round the clock customer support

- Convenient online submission

- Thorough peer review

- Inclusion in PubMed and all major indexing services

- Maximum visibility for your research

Submit your manuscript at www biomedcentral.com/submit

) Biomed Central 